\title{
The effect of traumatic occlusion on healing of periapical lesion: a case report
}

\begin{abstract}
The success of root canal treated teeth with periapical lesions have been reported in several studies. However, in some cases, such as traumatic occlusion, these lesions may remains table. On the other hand, the effect of traumatic occlusion in development of pulp and periapical diseases has not been known thoroughly. The article reports the remaining of periapical lesion after appropriate endodontic treatment and surgery. However, the patient symptoms improved after removal of premature contacts and periapical lesion healed.
\end{abstract}

Keywords: traumatic occlusion, periapical lesion healing

\author{
Volume 4 Issue 4 - 2016
}

\author{
Marziyeh Jafari,' Elaheh Moghim Farooji,' \\ Sara RamezanianNik ${ }^{2}$ \\ 'Department of Endodontic, Dental School, Mashhad University \\ of Medical Sciences, Iran \\ ${ }^{2}$ Department of Pediatrics Dentistry, Dental School, Mashhad \\ University of Medical Sciences, Iran
}

\begin{abstract}
Correspondence: Elaheh Moghim Farooji, Department of Endodontic, Dental School, Mashhad University of Medical Sciences, Iran, Tel 00985 I 3882950 I, Fax 00985 I38829500, Email marzieh_84@yahoo.com,moghim.elahe@yahoo.com
\end{abstract}

Received: June 07,2015 | Published: March 14, 2016

\section{Introduction}

The main reasons of pulp and periapical pathosis are microorganisms. Thereupon, many of periapical lesions may heal after normal treatment of the root canal and the reported successful rate by many of researches is $85-90 \%{ }^{1}$. However, sometimes periapical radiolucency abides because of traumatic occlusion, periodontal disease, leakage of restoration, overfilling and rare reasons such as systemic factors, and sometimes unknown reasons. ${ }^{2}$ When the intensity of produced force by occlusion exceed the ability of periodontium for resistance and distribution of these forces, occlusal trauma may extend and cause some changes in periodontal ligament, alveolar bone, cementum, and pulp. ${ }^{3}$ In absence of inflammation, traumatic occlusion can be eventuated to thermal sensitivity, increased mobility, periodontal ligament (PDL) widening, loss of crestal bone height and mass and root resorption, but not attachment loss. ${ }^{4,5}$ The effect of chronic occlusal trauma on progression of pulp and periodontal disease is unrecognized. It has been reported in animal experiments that inordinate occlusal force can cause inflammatory response, blood circulation disorder in periodontium, and pulp and also sensitize pulp nerves. ${ }^{6,7}$

Also, dentin hypersensitivity can be conceived by tiny premature occlusal contact $(<200 \mu \mathrm{m})$ without any special changes in periodontium. That can be the result of inflammation response and blood circulation disorder in pulp that can be removed by occlusal adjustment. ${ }^{6}$ It is specified in a study that one of the main factors in prolonging periapical healing of teeth which their root has been treated is occlusal trauma. ${ }^{8}$ Whereas, the result of short-term animal experiments has shown that occlusal trauma cause retardation in enlargement of periapical lesions. ${ }^{7}$ It is specified in Label study that there is strong correlation between premature occlusal contact on treated tooth in protrusive and working side movements and the existence of periapical radiolucency. ${ }^{8}$ It has been shown in other studies that occlusal forces can cause long-term apical inflammations and consequently can prevent healing. In a large percent of teeth with periapical lesions, occlusal force could accelerate and perpetuate periapical lesion. ${ }^{9,10}$ According to this fact that the connection between occlusal interference and unimproved periapical lesions is not precisely proved, the present case report demonstrates the influence of occlusal interference elimination on periradicular improvement in a patient after root-end surgery.

\section{Case report}

A 17 years old girl without any systemic disease and with pain and recurrent swelling in left maxillary molar was referred to the Department of Endodontics of Mashhad Faculty of Dentistry. According to transmittal report from surgery department, the patient had referred a year ago with swelling in left maxilla region and after getting panoramic radiography and observing radiolucent lesion in apical of distobuccal root in first maxillary molar, excisional surgery had been operated to definitive diagnosis and removed the lesion. The whole lesion had been sent to pathology department and an odontogenic infectious cyst had been distinguished, but not the type of cyst (Figure 1).

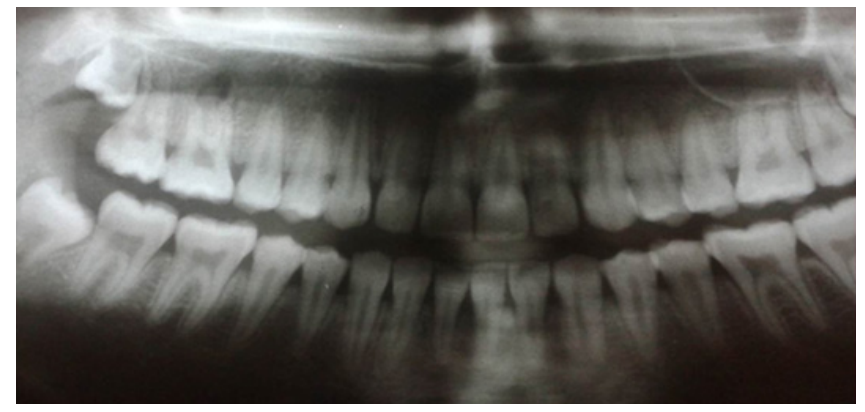

Figure I Panoramic view.

The patient had no problem in a year but after, swelling appeared to be in the same region. Therefore, she returned to surgery department for the second time and after getting another panoramic radiography, 
radiolucent lesion with mesial extension was observed. Biopsy had done and recognition was periapical granuloma. The reason for lesion extension was considered vast apical curettage following with a great possibility of losing vessels and nerves junctions in tooth and mistreatment of root after the first surgery; she was referred to endodontic department for treatment. Before that, vitality tests was done and the tooth had no response to cold, warm, and electric test (Pulp tester Parkell, USA) but in palpation and percussion tests, a little increased sensitivity than witness teeth was reported.

In order to endodontic treatment, the access cavity prepared, cleaning and shaping was done, calcium hydroxide (Golchadent, Karaj, Iran) was placed in canal by file and the coronal seal was achieved by temporary restoration (Ariadent Coltosol, Tehran, Iran). After 10 days of placing calcium hydroxide, the patient came back for continuing the treatment. All the symptoms were improved and she had no complaint about pain or swelling through these 10 days. The treatment was over and amalgam (World work, Vicenza, Italy) was done to establish coronal seal and occlusal contacts were checked during that session (Figure 2) \& (Figure 3).

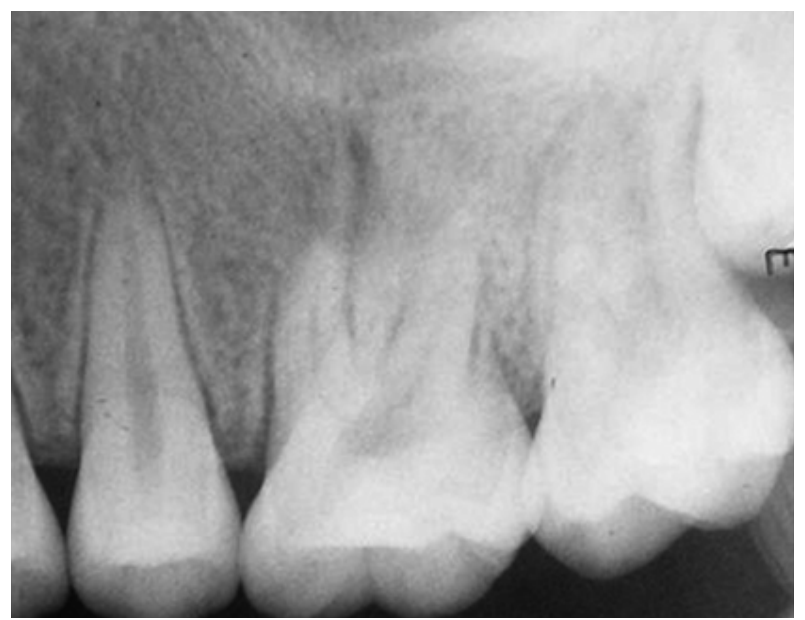

Figure 2 Initial radiography.

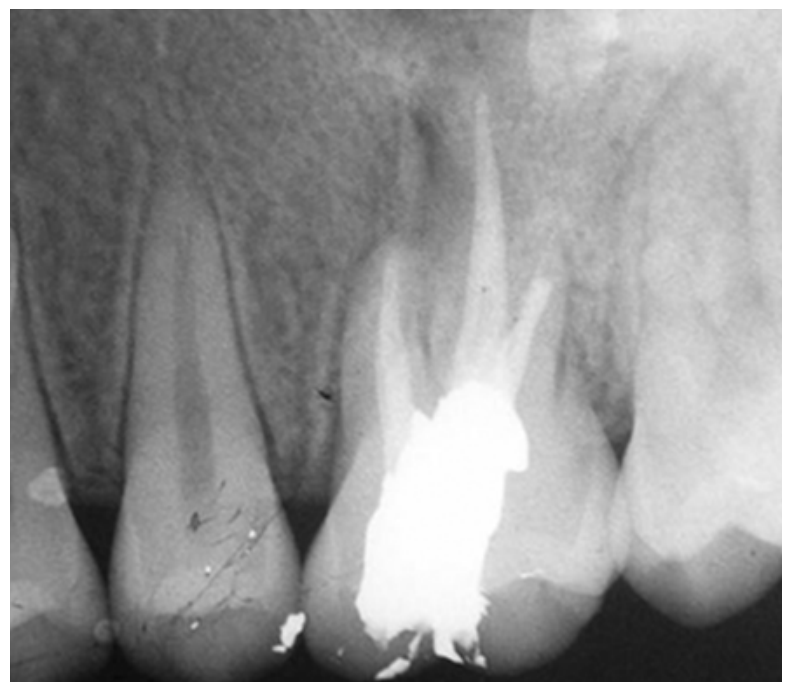

Figure 3 Radiograph taken immediately after root canal therapy and Amalgam Filling.

In 1 month and 3 month follow up, clinical symptoms were normal but in 6 month follow up after the treatment, the patient announced existence of a fistula since a month before that with tracing, it was understood that it is related to mesiobuccal the root of the first molar (Figure 4A \& 4B). Because of a proper and ideal root treatment, apical surgery was done without any root retreatment. After retracting the flap, two bone deficiencies in apical of mesiobuccal and distobuccal root were observed. The curettage was done on granulation tissue and after rinsing with saline (Samen, Mashhad, Iran); the lesion region was filled by bone powder (Conebone, Kish free zone, Iran) and was sutured. The patient came back in a week after surgery to pull out the sutures and improvement symptoms were observed.

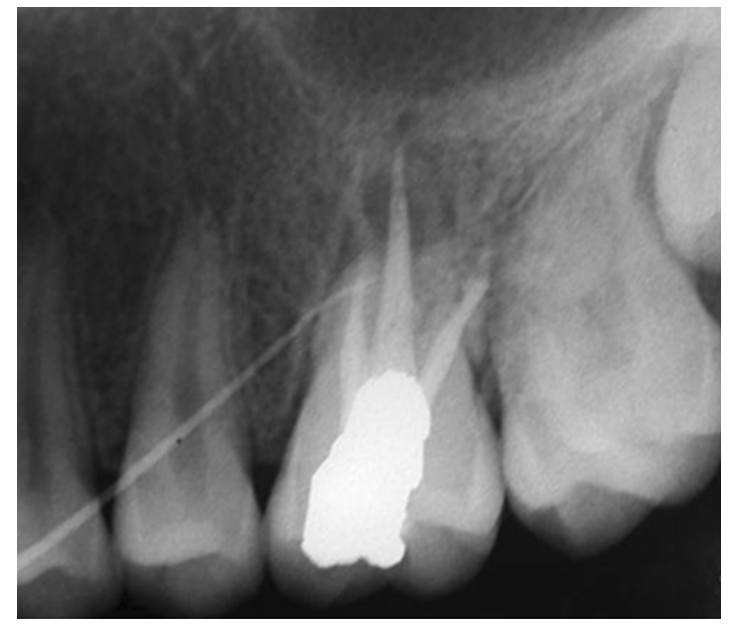

Figure 4A Radiograph taken immediately after root canal therapy and Amalgam Filling.

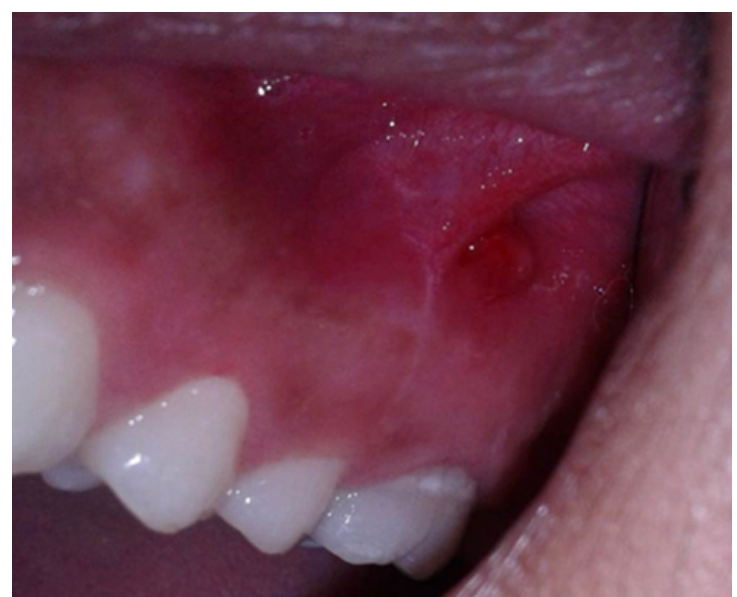

Figure 4B Radiograph taken 6 months after treatment and tracing with Gutta percha, Photograph.

In 1 month follow up after the second surgery; sinus tract sinus was not improved (Figure 5). The patient's occlusion was rechecked and a small premature contact was removed in palatal cusp of buccal slope. It was explained to the patient for doing retreatment in future session. In the next session which was 5 months later because of the patients unreturning, the improvement of sinus tract was observed and it could not be traced. The patient did not report any problem and in radiography consideration, the size of lesion was decreased (Figure 6). Occlusal contacts were rechecked and the tooth was completely removed from occlusion. In 10 days follow up and 1 year clinical recovery was observed (Figure 7). 


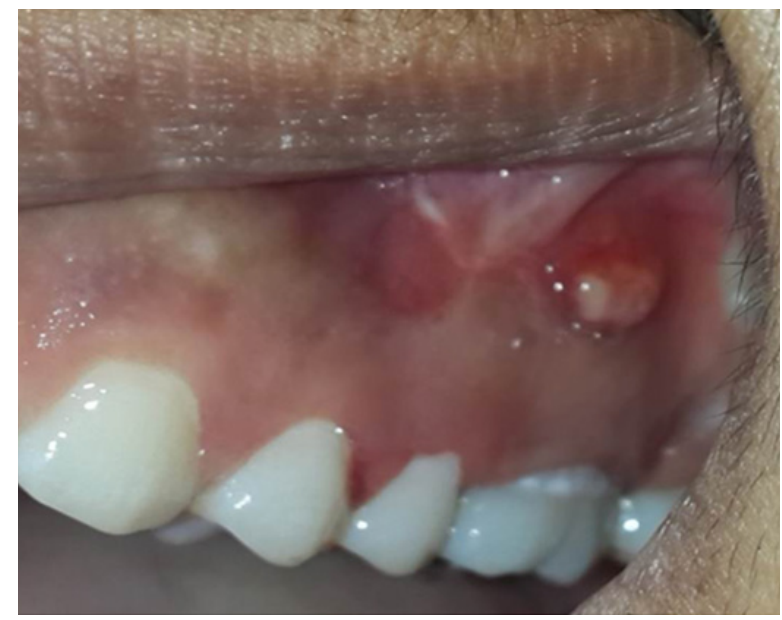

Figure 5 Photograph taken I month after surgery.

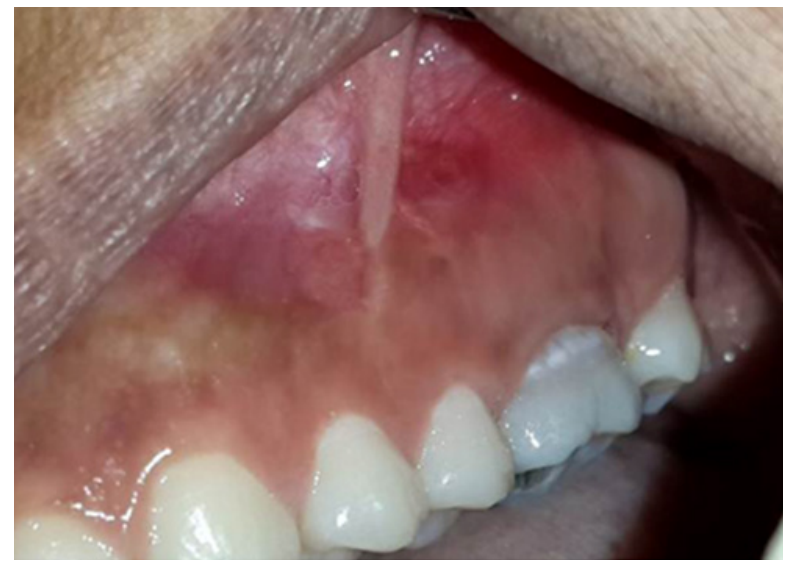

Figure 6 Photograph taken 5 month after surgery.

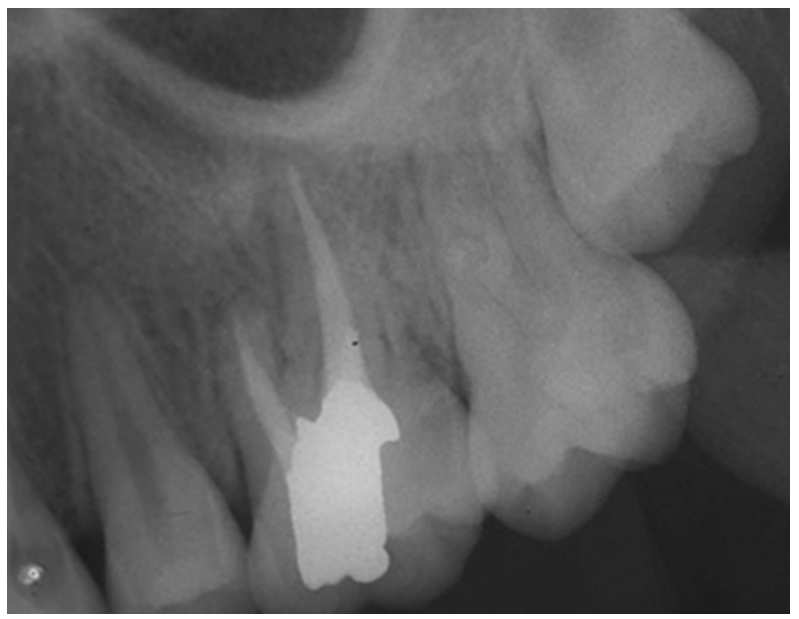

Figure 7 Radiograph taken I year after surgery.

\section{Discussion}

After the proper non-surgical root canal treatment, teeth with apical periodontitis regardless that whether bacterial culture before filling is negative or not, will be improved in $87 \%$ of cases. Therefore, it is supposed that some of cysts especially pocket cysts will be improved after the non-surgical treatment. True cysts, because of their self-sustaining nature, are less possible for them to improve after non-surgical root canal treatment, so surgery is essential. ${ }^{11}$ The reported successful rate of endodontic surgery in several articles is $37-91 \%$. According to the most dissertations, the most important predictable factors in success of surgery treatment are the patient age, lesion size before the surgery, apical and coronal seal and the length of root filling. ${ }^{11}$ Healing mechanism of periapical wound after nonsurgical treatment and endodontic surgery is completely the same, but the kinetics of healing of periapical wound after endodontic surgery are much faster than non-surgical endodontic treatment. ${ }^{12}$ In several studies, it is said that the reasons for stability of periapical lesions are the extra radicular Enterococcus faecalis infection, the overextension of filling material, the existence of cholesterol crystals, root fracture, traumatic occlusion and some systemic diseases. ${ }^{12}$ The occlusal trauma effect is less-known in pulp and periapical fibers and especially in root treatments results. Traumatic occlusion is related to periapical osteosclerosis or over-mineralization of bone around vital tooth without any sign. ${ }^{8}$

Traumatic occlusion will have one or more radiographic and clinical symptoms, containing mobility, pain in chewing and percussion, fremitus, occlusal prematurities/discrepancies, facet existence, tooth migration, chipped or fractured teeth, thermal sensitivity, PDL widening, bone loss and root resorption. ${ }^{13}$ However it is not proved that the existence of premature occlusal contacts can create new periapical lesions or perpetuate the former lesions, but the present report demonstrate that the existence of occlusal trauma can cause un-improvement of lesions even after the surgery. In conclusion, it is important to assess and remove premature contacts in tooth through the treatment and definitely it is one of the most effective factors in treatment results.

\section{Funding}

None.

\section{Acknowledgements}

None.

\section{Conflicts of interest}

The author declares there are no conflicts of interest.

\section{References}

1. Nair PN. New perspectives on radicular cysts: do they heal? A Review. Int Endod J. 1998;31(3):155-160.

2. Bergenholtz G, Horsted-Bindslev P, Reit C. Textbook of Endodontology. $2^{\text {nd }}$ ed. 2009. p. 45.

3. Mic Newman M, Takei H, Klokkevold P. Carranza's Clinical Periodontology. $11^{\text {th }}$ ed. 2011. p. 215.

4. Hallmon WW. Occlusal trauma: effect and impact on the periodontium. Ann Periodontol. 1999;4(1):2-8.

5. American Academy of Periodontology. Parameter on occlusal traumatism in patients with chronic periodontitis. Parameters of Care J Periodontol. 2000;71 (Suppl 5):873-875.

6. Ikeda T, Nakano M, Bando E. The effect of light premature occlusal contact on tooth pain threshold in humans. J Oral Rehabil. 1998;25(8):589-595.

7. Kumazawa M, Kohsaka T, Yamasaki M. Effect of Traumatic Occlusion on Periapical Lesions in Rats. $J$ Endod. 1995;21(7):372-376. 
8. Iqbal MK, Johansson AA, Akeel RF. A Retrospective Analysis of Factors Associated with the Periapical Status of Restored, Endodontically Treated Teeth. Int J Prosth. 2003;16(1):31-39.

9. Matsumoto T, Nagai T, Ida K. Factors affecting successful prognosis of root canal treatment. J Endod. 1987;13(5):239-242.

10. Grossman LI, Schilder H. Periodontal-endodontal therapy. In: Endodontic Practice. $10^{\text {th }}$ ed. Lea \& Febiger, Philadelphia, USA; 1981. p. 384-398.
11. Song M, Jung IY, Lee SJ. Prognostic Factors for Clinical Outcomes in Endodontic Microsurgery: A Retrospective Study. $J$ Endod. 2011;37(7):927-933.

12. Hargreaves k, Cohen S, Berman L. Cohen's pathways of the pulp, St. Louis, USA: Mosby; 2011. 551 p.

13. Commander R. Trauma from occlusion: a review. Clin Update. 2004;26(1):25-27. 